\title{
Verification of Some Properties of the C-nilpotent Multiplier in Lie Algebras
}

\section{Sadeghieh A, Araskhan M*}

Yazd Branch, Islamic Azad University, Iran

\begin{abstract}
The purpose of this paper is to obtain some inequalities and certain bounds for the dimension of the c-nilpotent multiplier of finite dimensional nilpotent Lie algebras and their factor Lie algebras. Also, we give an inequality for the dimension of the c-nilpotent multiplier of $L$ connected with dimension of the Lie algebras $\gamma_{d}(L)$ and $L / Z_{d-1}(L)$. Finally, we compare our results with the previously known result.
\end{abstract}

Keywords: C-nilpotent multiplier; Nilpotent lie algebra; Lie algebra

\section{Introduction}

All Lie algebras referred to in this article are (of finite or infinite dimension) over a fixed field $\mathrm{F}$ and the square brackets [, ] denotes the Lie product. Let $0 \rightarrow \mathrm{R} \rightarrow \mathrm{F} \rightarrow \mathrm{L} \rightarrow 0$ be a free presentation of a Lie algebra $\mathrm{L}$, where $\mathrm{F}$ is a free Lie algebra. Then we define the, c-nilpotent multiplier $c \geq 1$, to be

$$
\mathrm{M}^{(\mathrm{c})}(\mathrm{L})=\frac{\left(\mathrm{R} \bigcap \gamma_{\mathrm{c}+1}(\mathrm{~F})\right)}{\gamma_{\mathrm{c}+1}(\mathrm{R}, \mathrm{F})},
$$

where $\gamma_{c+1}(F)$ is the $(c+1)$-th term of the lower central series of $F, \gamma_{1}(R$, $\mathrm{F})=1$ and $\gamma_{\mathrm{c}+1}(\mathrm{R}, \mathrm{F})=\left[\gamma_{\mathrm{c}}(\mathrm{R}, \mathrm{F}), \mathrm{F}\right]$. This is analogous to the definition of the Baer-invariant of a group with respect to the variety of nilpotent groups of class at most $\mathrm{c}$ given by Baer [1-3] (for more information on the Baer invariant of groups). The Lie algebra $\mathrm{M}^{(1)}(\mathrm{L})=\left(\mathrm{R} \cap \mathrm{F}^{2}\right) /[\mathrm{R}, \mathrm{F}]=\mathrm{M}(\mathrm{L})$ is the most studied Schur multiplier of $\mathrm{L}[4,5]$. It is readily verified that the Lie algebra $\mathrm{M}^{(\mathrm{c})}(\mathrm{L})$ is abelian and independent of the choice of the free presentation of L [6]. The purpose of this paper is to obtain some inequalities for the dimension of the c-nilpotent multiplier of finite dimensional nilpotent Lie algebras and their factor Lie algebras (Corollary 2.3 and Corollary 2.5). Finally, we compare our results to upper bound given [6]. First, we show that for each ideal $\mathrm{N}$ in $\mathrm{L}$, there is a close relationship between the $\mathrm{M}^{(\mathrm{c})}(\mathrm{L})$ and $\mathrm{M}^{(\mathrm{c})}(\mathrm{L} / \mathrm{N})$

Lemma 1.1. Let $\mathrm{L}$ be Lie algebra with a free presentation $0 \rightarrow R \rightarrow F \rightarrow L \rightarrow 0$. If $S$ is an ideal in $F$ with $N \cong S / R$, then the following sequences are exact:

(i) $0 \rightarrow \frac{\mathrm{R} \cap \gamma_{\mathrm{c}+1}(\mathrm{~S}, \mathrm{~F})}{\gamma_{\mathrm{c}+1}(\mathrm{R}, \mathrm{F})} \rightarrow \mathrm{M}^{(\mathrm{c})}(\mathrm{L}) \rightarrow \mathrm{M}^{(\mathrm{c})}(\mathrm{L} / \mathrm{N}) \rightarrow \frac{\mathrm{N} \cap \gamma_{\mathrm{c}+1}(\mathrm{~L})}{\gamma_{\mathrm{c}+1}(\mathrm{~N}, \mathrm{~L})} \rightarrow 0$,

(ii) $0 \rightarrow(\mathrm{N})_{\mathrm{ab}} \otimes^{\mathrm{c}}\left(\mathrm{L} / \mathrm{L}^{2}\right)_{\mathrm{ab}} \rightarrow \mathrm{M}^{(\mathrm{c})}(\mathrm{L}) \rightarrow \mathrm{M}^{(\mathrm{c})}(\mathrm{L} / \mathrm{N}) \rightarrow \mathrm{N} \cap \gamma_{\mathrm{c}+1}(\mathrm{~L}) \rightarrow 0$.

under the condition that $\mathrm{N}$ is central, $\mathrm{N} \otimes^{\mathrm{c}} \mathrm{M}=\mathrm{N} \otimes \underbrace{\mathrm{M} \otimes \ldots \otimes \mathrm{M}}$ and $(\mathrm{N})_{\mathrm{ab}}=\mathrm{N} / \mathrm{N}^{2}$.

Proof. We prove only part (ii). Since N is central, $[\mathrm{S}, \mathrm{F}] \subseteq \mathrm{R}$ and

$\gamma_{\mathrm{c}+1}\left(\mathrm{~S}, \mathrm{~F}^{2}+\mathrm{R}\right) \subseteq \gamma_{\mathrm{c}+1}(\mathrm{~S}, \mathrm{R})+\gamma_{\mathrm{c}+1}\left(\mathrm{R}, \mathrm{F}^{2}\right) \subseteq \gamma_{\mathrm{c}+1}(\mathrm{R}, \mathrm{F})+\gamma_{\mathrm{c}+1}(\mathrm{R},[\mathrm{F}, \mathrm{F}]) \subseteq \gamma_{\mathrm{c}+1}(\mathrm{R}, \mathrm{F})$

Now, we have the following homomorphism

$\alpha: S / R \otimes^{c} F /\left(F^{2}+R\right) \rightarrow \frac{R \cap \gamma_{c+1}(F)}{\gamma_{c+1}(R, F)}$

such that $\operatorname{Im} \alpha=\gamma_{c+1}(S, F) / \gamma_{c+1}(R, F)$. Now the result holds by part (i).
The following corollary is an immediate consequence of Lemma 1.1, which gives some elementary results about dimension of the c-nilpotent multiplier of finite dimensional Lie algebras see corollary 2.2 of Salemkar et al. [6].

Corollary 1.2. Let $\mathrm{N}$ be an ideal of Lie algebra L. Then

(i) $\operatorname{dim} \mathrm{M}^{(\mathrm{c})}(\mathrm{L})+\operatorname{dim}\left(\mathrm{N} \cap \gamma_{\mathrm{c}+1}(\mathrm{~L})\right)=\operatorname{dim} \mathrm{M}^{(\mathrm{c})}(\mathrm{L} / \mathrm{N})+\operatorname{dim}\left(\frac{\gamma_{\mathrm{c}+1}(\mathrm{~S}, \mathrm{~F})}{\gamma_{\mathrm{c}+1}(\mathrm{R}, \mathrm{F})}\right)$,

Where F,S,R are defined in Lemma 1.1.

(ii) $\operatorname{dim} \mathrm{M}^{(\mathrm{c})}(\mathrm{L})+\operatorname{dim}\left(\mathrm{N} \cap \gamma_{\mathrm{c}+1}(\mathrm{~L})\right) \leq \operatorname{dim} \mathrm{M}^{(\mathrm{c})}(\mathrm{L} / \mathrm{N})+\operatorname{dim}\left((\mathrm{N})_{\mathrm{ab}} \otimes^{\mathrm{c}}\left(\mathrm{L} / \mathrm{L}^{2}\right)_{\mathrm{ab}}\right)$

Suppose that $\mathrm{L}$ is generated by $\mathrm{n}$ elements. Let $\mathrm{F}$ be a free Lie algebra generated by $\mathrm{n}$ elements and $\mathrm{L} \cong \mathrm{F} / \mathrm{R}$ Witt,s formula from Bahturin et al. [7] gives us

$$
\operatorname{Dim} \gamma_{d}(F) / \gamma_{d+1}(F)=\frac{1}{d} \sum_{m \mid d} \mu(m) n^{\frac{d}{m}}=1_{n}(d),
$$

where $\mu(\mathrm{m})$ is the Mobius function, defined by $\mu(1)=1, \mu(\mathrm{k})=0$ if $\mathrm{K}$ is divisible by a square, and $\mu\left(\mathrm{p}_{1} \ldots \mathrm{p}_{\mathrm{s}}\right)=(-1)^{\mathrm{s}}$ if $\mathrm{p}_{1}, \ldots, \mathrm{p}_{\mathrm{s}}$ are distinct prime numbers.

Lemma 1.3. Let $\mathrm{L}$ be an abelian Lie algebra of dimension $\mathrm{n}$. Then $\operatorname{dim} \mathrm{M}^{(\mathrm{c})}(\mathrm{L})=1_{\mathrm{n}}(\mathrm{c}+1)$. In particular, $\operatorname{dim} \mathrm{M}(\mathrm{L})=\frac{1}{2} \mathrm{n}(\mathrm{n}-1)$.

Proof. Consider a free Lie algebra F freely generated by $\mathrm{n}$ elements. By Witt's formula, $\mathrm{F} / \mathrm{F}^{2}$ is an abelian Lie algebra of dimension $\mathrm{n}$, and so it is isomorphic to L. Hence $\operatorname{dim} \mathrm{M}^{(\mathrm{c})}(\mathrm{L})=\operatorname{dim}\left(\gamma_{\mathrm{c}+1}(\mathrm{~F}) / \gamma_{\mathrm{c}+2}(\mathrm{~F})\right)$, which gives the result.

Let $\mathrm{L}=\gamma_{1}(\mathrm{~L}) \supset \gamma_{2}(\mathrm{~L}) \supset \ldots \supset \gamma_{\mathrm{c}}(\mathrm{L}) \supset \gamma_{\mathrm{c}+1}(\mathrm{~L})=0$ be the lower central series of nilpotent Lie algebra, $\mathrm{L}$. $\mathrm{L}$ is said to have class $\mathrm{c}$ if $\mathrm{c}$ is the least integer for which $\gamma_{c+1}(L)=0$. Furthermore, if $\operatorname{dim} \gamma_{j}(L) / \gamma_{j+1}(L)=1$ for $j=2,3, \ldots, c$ and $\operatorname{dim} L / \gamma_{2}(L)=2$, then $L$ is said to be of maximal class c. Additionally, let $0=\mathrm{Z}_{0}(\mathrm{~L}) \subset \mathrm{Z}_{1}(\mathrm{~L}) \subset \mathrm{Z}_{2}(\mathrm{~L}) \subset \ldots \subset \mathrm{Z}_{\mathrm{c}}(\mathrm{L})=\mathrm{L}$ be the upper central series of nilpotent Lie algebra $\mathrm{L}$. If $\mathrm{L}$ is of maximal

*Corresponding author: Araskhan M, Yazd Branch, Islamic Azad University, Iran Tel: +982147911; E-mail: h.araskhan@yahoo.com

Received April 21, 2015; Accepted July 20, 2015; Published July 29, 2015

Citation: Sadeghieh A, Araskhan M (2015) Verification of Some Properties of the C-nilpotent Multiplier in Lie Algebras. J Generalized Lie Theory Appl 9: 228 doi:10.4172/1736-4337.1000228

Copyright: @ 2015 Sadeghieh A, et al. This is an open-access article distributed under the terms of the Creative Commons Attribution License, which permits unrestricted use, distribution, and reproduction in any medium, provided the original author and source are credited. 
class, then $Z_{\mathrm{i}}(\mathrm{L})=\gamma_{\mathrm{c}-\mathrm{i}+1}(\mathrm{~L})$ for $0 \leq \mathrm{i} \leq \mathrm{c}$.

By the above notation we have the following corollary.

Corollary 1.4. Let $\mathrm{L}$ be a finite dimensional nilpotent Lie algebra of maximal class $(\mathrm{c}+1)$, then

$\operatorname{dim} \mathrm{M}^{(\mathrm{c})}(\mathrm{L}) \leq \operatorname{dim} \mathrm{M}^{(\mathrm{c})}(\mathrm{L} / \mathrm{Z}(\mathrm{L}))+2^{\mathrm{c}}-1$

Proof. Using Corollary 1.2(ii) with $\mathrm{N}=\mathrm{Z}(\mathrm{L})$, we get

$\operatorname{dim} \mathrm{M}^{(\mathrm{c})}(\mathrm{L})+\operatorname{dim} \mathrm{Z}(\mathrm{L})=\operatorname{dim} \mathrm{M}^{(\mathrm{c})}(\mathrm{L})+1 \leq \operatorname{dim}^{(\mathrm{c})}(\mathrm{L} / \mathrm{Z}(\mathrm{L}))+(1) 2^{\mathrm{c}}$.

\section{Discussion and Results}

\section{Bounds on $\operatorname{dim} \mathbf{M}^{(\mathrm{c})}(\mathbf{L})$}

Let $\mathrm{L}$ be a finite dimensional nilpotent Lie algebra of class $\mathrm{d} \geq$ 2. First, we give an inequality for the dimension of the c-nilpotent multiplier of $L$ connected with dimension of the Lie algebras $\gamma_{d}(L)$ and $\mathrm{L} / \mathrm{Z}_{\mathrm{d}-1}(\mathrm{~L})$ (Corollary 2.3) and some inequalities for the dimension of the $\mathrm{c}$-nilpotent multiplier of finite dimensional nilpotent Lie algebras will be given. For this purpose, we need the following two lemmas.

Lemma 2.1. Let $\mathrm{H}$ and $\mathrm{N}$ be ideals of Lie algebra $\mathrm{L}$ and $\mathrm{N}=\mathrm{N}_{0} \supseteq \mathrm{N}_{1} \supseteq \ldots$, a chain of ideals of $\mathrm{N}$ such that $\left[\mathrm{N}_{\mathrm{i}}, \mathrm{L}\right] \subseteq \mathrm{N}_{\mathrm{i}+1}$ for all $\mathrm{I}=1,2, \ldots$. Then

$\left[\mathrm{N}_{\mathrm{i}},\left[\mathrm{H},{ }_{\mathrm{j}} \mathrm{L}\right]\right] \subseteq \mathrm{N}_{\mathrm{i}+\mathrm{j}+1}$ for all $\mathrm{i}, \mathrm{j}$.

Proof. We have

$$
\begin{aligned}
{\left[N_{i},\left[H,{ }_{j+1} L\right]\right] } & \subseteq\left[N_{i},\left[\left[H,{ }_{j} L\right], L\right]\right] \\
& \subseteq\left[\left[N_{i},\left[H,{ }_{j} L\right]\right], L\right]+\left[\left[N_{i}, L\right],\left[H,{ }_{j} L\right]\right] \\
& \subseteq\left[N_{i+j+1}, L\right]+\left[N_{i+1},\left[H,{ }_{j} L\right]\right] \\
& \subseteq N_{i+j+2}+N_{i+j+2}=N_{i+j+2} .
\end{aligned}
$$

Now, the assertion follows by induction on $j$.

Lemma 2.2. Let $\mathrm{L}$ be a finite dimensional nilpotent Lie algebra of class $\mathrm{d} \geq 2$. Let $0 \rightarrow \mathrm{R} \rightarrow \mathrm{F} \rightarrow \mathrm{L} \rightarrow 0$ be a free presentation of $\mathrm{L}$, then $\frac{\gamma_{c+1}\left(\gamma_{d}(F)+R, F\right)}{\gamma_{c+1}(R, F)}$ is a homomorphic image of $\gamma_{d}(L) \otimes \underbrace{\frac{L}{Z_{d-1}(L)} \otimes \cdots \otimes \frac{L}{Z_{d-1}(L)}}_{c \text {-times }}$.

Proof. Put $\mathrm{Z}_{\mathrm{k}}(\mathrm{L})=\mathrm{T}_{\mathrm{k}} / \mathrm{R}$ for $0 \leq \mathrm{k} \leq \mathrm{d}$. Now consider the following chain

$\mathrm{S}=\mathrm{T}_{\mathrm{d}} \supseteq \cdots \supseteq \mathrm{T}_{\mathrm{k}} \supseteq \mathrm{T}_{\mathrm{k}-1} \supseteq \cdots \supseteq \mathrm{T}_{1} \supseteq \mathrm{T}_{0}=\mathrm{R}$.

Since $\left[\mathrm{T}_{\mathrm{k}}, \mathrm{F}\right] \subseteq \mathrm{T}_{\mathrm{k}-1}$, then by Lemma 2.1,

$\left[\mathrm{T}_{\mathrm{d}-1},\left[\gamma_{\mathrm{d}-2}(\mathrm{~F}), \mathrm{F}\right]\right] \subseteq \mathrm{T}_{\mathrm{d}-1-(\mathrm{d}-2+1)}=\mathrm{T}_{0}=\mathrm{R}$.

Therefore,

$$
\begin{aligned}
& {[\gamma_{d}(F)+R, \underbrace{T_{d-1}, \ldots, T_{d-1}}_{c-\text { times }}] \subseteq[\gamma_{d}(F), \underbrace{T_{d-1}, \ldots, T_{d-1}}_{c-\text { times }}]+[R, \underbrace{T_{d-1}, \ldots, T_{d-1}}_{c-\text { times }}]} \\
& \subseteq[\gamma_{d}(F), \underbrace{T_{d-1}, \ldots, T_{d-1}}_{c-1 \text { times }}]+\gamma_{c+1}(R, F) \\
& \subseteq[\left[\left[T_{d-1}, F\right], \gamma_{d-1}(F)\right], \underbrace{T_{d-1}, \ldots, T_{d-1}}_{(c-1)-\text { times }}] \\
& +[\left[\left[T_{d-1},\left[\gamma_{d-2}(F), F\right]\right], F\right], \underbrace{T_{d-1}, \ldots, T_{d-1}}_{(c-1)-\text { times }}]+\gamma_{c+1}(R, F)
\end{aligned}
$$

$$
\begin{aligned}
& \subseteq[\left[T_{d-2}, \gamma_{d-1}(F)\right], \underbrace{T_{d-1}, \ldots, T_{d-1}}_{(c-1) \text {-times }}]+\gamma_{c+1}(R, F) \\
& \vdots \\
& \subseteq[\left[T_{0}, \gamma_{1}(F)\right], \underbrace{T_{d-1}, \ldots, T_{d-1}}_{(c-1)-\text { times }}]+\gamma_{c+1}(R, F) \\
& \subseteq \gamma_{c+1}(R, F) .
\end{aligned}
$$

The latter inclusion gives the following epimorphism

$$
\frac{\gamma_{d}(F)+R}{R} \times \underbrace{\frac{F}{T_{d-1}} \times \cdots \frac{F}{T_{d-1}}}_{c \text {-times }} \rightarrow \frac{\gamma_{c+1}\left(\gamma_{d}(F)+R, F\right)}{\gamma_{c+1}(R, F)}
$$$$
\left(x+R, f_{1}+T_{d-1}, \ldots, f_{c}+T_{d-1}\right) \mapsto\left[x, f_{1}, \ldots, f_{c}\right]+\gamma_{c+1}(R, F) .
$$

Corollary 2.3. Under the assumptions and notation of the above Lemma, we have

$$
\operatorname{dim} M^{()}(L)+\operatorname{dim}\left(\gamma_{d}(L)+\gamma_{c+1}(L)\right) \leq \operatorname{dim} M^{(c)}\left(L / \gamma_{d}(L)\right)+
$$
$\operatorname{dim} \gamma_{d}(L)\left[\operatorname{dim}\left(\frac{L}{Z_{d-1}(L)}\right)\right]^{c}$. Proof. In Corollary 1.2(i), taking $\mathrm{N}=\gamma_{\mathrm{d}}(\mathrm{L})=\frac{\gamma_{\mathrm{d}}(\mathrm{F})+\mathrm{R}}{\mathrm{R}}$. Now by
Lemma 2.2, we have

$$
\begin{aligned}
& \operatorname{dim} \mathrm{M}^{(\mathrm{c})}(\mathrm{L})+\operatorname{dim}\left(\gamma_{\mathrm{d}}(\mathrm{L})+\gamma_{\mathrm{c}+1}(\mathrm{~L})\right)=\operatorname{dim} \mathrm{M}^{(\mathrm{c})}\left(\mathrm{L} / \gamma_{\mathrm{d}}(\mathrm{L})\right)+\operatorname{dim}\left(\frac{\gamma_{\mathrm{c}+1}\left(\gamma_{\mathrm{d}}(\mathrm{F})+\mathrm{R}, \mathrm{F}\right)}{\gamma_{\mathrm{c}+1}(\mathrm{R}, \mathrm{F})}\right) \\
& \leq \operatorname{dim}(\gamma_{d}(L) \otimes \underbrace{\frac{L}{Z_{d-1}(L)} \otimes \cdots \otimes \frac{L}{Z_{d-1}(L)}}_{c-\text { times }}) \\
& +\operatorname{dim} \mathrm{M}^{(\mathrm{c})}\left(\mathrm{L} / \gamma_{\mathrm{d}}(\mathrm{L})\right) \\
& =\operatorname{dim} \mathrm{M}^{(\mathrm{c})}\left(\mathrm{L} / \gamma_{\mathrm{d}}(\mathrm{L})\right)+\operatorname{dim} \gamma_{\mathrm{d}}(\mathrm{L})\left[\operatorname{dim}\left(\frac{\mathrm{L}}{Z_{\mathrm{d}-1}(\mathrm{~L})}\right)\right]^{\mathrm{c}} .
\end{aligned}
$$

In following, we give another an inequality for the dimension of the c-nilpotent multiplier of finite dimensional nilpotent Lie algebras.

Theorem 2.4. Let $\mathrm{L}$ be a finite dimensional nilpotent Lie algebra of class $\geq 1$, then

$\operatorname{dim} M^{(c)}(L) \leq \operatorname{dim} M^{(c)}\left(L / L^{2}\right)+\operatorname{dim} L^{2}\left[\operatorname{dim}(L / Z(L))-\operatorname{dim}(L / Z(L))^{2}\right]^{c}-\operatorname{dim}\left(\gamma_{c+1}(L)\right)$.

Proof. We use induction on the class of $\mathrm{L}$. If $\mathrm{L}$ is of class 1 , then $\mathrm{L}^{2}=0$ and the result holds. Assume the result for nilpotent Lie algebras of class to be less than $\mathrm{d}$ and let $\mathrm{L}$ have class $\mathrm{m}=$ d-1. Note that $\gamma_{\mathrm{m}}(\mathrm{L}) \subseteq \mathrm{Z}(\mathrm{L}), \mathrm{L}^{2} \subseteq \mathrm{Z}_{\mathrm{m}-1}(\mathrm{~L}),\left(\mathrm{L} / \gamma_{\mathrm{m}}(\mathrm{L})\right)^{2}=\mathrm{L}^{2} / \gamma_{\mathrm{m}}(\mathrm{L})$ and $\mathrm{Z}(\mathrm{L}) / \gamma_{\mathrm{m}}(\mathrm{L}) \subseteq \mathrm{Z}\left(\mathrm{L} / \gamma_{\mathrm{m}}(\mathrm{L})\right)$. Forconvenience, let $\mathrm{A}=\left(\mathrm{L} / \gamma_{\mathrm{m}}(\mathrm{L})\right) / \mathrm{Z}\left(\mathrm{L} / \gamma_{\mathrm{m}}(\mathrm{L})\right)$ and $\mathrm{B}=\mathrm{L} / \mathrm{Z}(\mathrm{L})=\left(\mathrm{L} / \gamma_{\mathrm{m}}(\mathrm{L})\right) /\left(\mathrm{Z}(\mathrm{L}) / \gamma_{\mathrm{m}}(\mathrm{L})\right)$. Since $\mathrm{A}$ is a homomorphic image of $\mathrm{B}$, it follows that $\operatorname{dim} \mathrm{A} / \mathrm{A}^{2} \leq \operatorname{dim} \mathrm{B} / \mathrm{B}^{2}$. By induction,

$$
\begin{aligned}
& \operatorname{dimM}(\mathrm{c})\left(\mathrm{L} / \gamma_{\mathrm{m}}(\mathrm{L})\right) \leq \operatorname{dimM}^{(\mathrm{c})}\left(\left(\mathrm{L} / \gamma_{\mathrm{m}}(\mathrm{L})\right) /\left(\mathrm{L} / \gamma_{\mathrm{m}}(\mathrm{L})\right)^{2}\right) \\
& +\operatorname{dim}\left(\mathrm{L} / \gamma_{\mathrm{m}}(\mathrm{L})\right)^{2}\left[\operatorname{dim}\left(\mathrm{A} / \mathrm{A}^{2}\right)\right]^{\mathrm{c}}-\operatorname{dim}\left(\gamma_{\mathrm{c}+1}\left(\mathrm{~L} / \gamma_{\mathrm{m}}(\mathrm{L})\right)\right) \\
& \leq \operatorname{dimM}^{(\mathrm{c})}\left(\mathrm{L} / \mathrm{L}^{2}\right)+\operatorname{dim}\left(\mathrm{L}^{2} / \gamma_{\mathrm{m}}(\mathrm{L})\right)\left[\operatorname{dim}\left(\mathrm{B} / \mathrm{B}^{2}\right)\right]^{\mathrm{c}}-\operatorname{dim}\left(\gamma_{\mathrm{c}+1}\left(\mathrm{~L} / \gamma_{\mathrm{m}}(\mathrm{L})\right)\right) .
\end{aligned}
$$

By Corollary 2.3,

$$
\begin{aligned}
& \operatorname{dimM}^{(\mathrm{c})}(\mathrm{L}) \leq \operatorname{dimM}^{(\mathrm{c})}\left(\mathrm{L} / \gamma_{\mathrm{m}}(\mathrm{L})\right)+\operatorname{dim}\left(\gamma_{\mathrm{m}}(\mathrm{L})\right)\left[\operatorname{dim}\left(\mathrm{L} / \mathrm{Z}_{\mathrm{m}-1}(\mathrm{~L})\right)\right]^{\mathrm{c}} \\
& -\operatorname{dim}\left(\gamma_{\mathrm{m}}(\mathrm{L}) \cap \gamma_{\mathrm{c}+1}(\mathrm{~L})\right) . \\
& \text { Also, } \operatorname{dim}\left(\mathrm{L} / \mathrm{Z}_{\mathrm{m}-1}(\mathrm{~L})\right) \leq \operatorname{dim}\left(\mathrm{L} /\left(\mathrm{L}^{2}+\mathrm{Z}(\mathrm{L})\right)\right)=\operatorname{dim}\left(\mathrm{B} / \mathrm{B}^{2}\right)
\end{aligned}
$$


Therefore,

$$
\begin{aligned}
& \operatorname{dimM}^{(\mathrm{c})}(\mathrm{L}) \leq \operatorname{dim} \mathrm{M}^{(\mathrm{c})}\left(\mathrm{L} / \mathrm{L}^{2}\right)+\operatorname{dim}\left(\mathrm{L}^{2} / \gamma_{\mathrm{m}}(\mathrm{L})\right)\left[\operatorname{dim}\left(\mathrm{B} / \mathrm{B}^{2}\right)\right]^{\mathrm{c}}-\operatorname{dim}\left(\gamma_{\mathrm{c}+1}\left(\mathrm{~L} / \gamma_{\mathrm{m}}(\mathrm{L})\right)\right) \\
& +\operatorname{dim}\left(\gamma_{\mathrm{m}}(\mathrm{L})\right)\left[\operatorname{dim}\left(\mathrm{B} / \mathrm{B}^{2}\right)\right]^{\mathrm{c}}-\operatorname{dim}\left(\gamma_{\mathrm{m}}(\mathrm{L}) \cap \gamma_{\mathrm{c}+1}(\mathrm{~L})\right) \\
& \leq \operatorname{dim} \mathrm{M}^{(\mathrm{c})}\left(\mathrm{L} / \mathrm{L}^{2}\right)+\operatorname{dim} \mathrm{L}^{2}\left[\operatorname{dim}\left(\mathrm{B} / \mathrm{B}^{2}\right)\right]^{\mathrm{c}}-\operatorname{dim}\left(\gamma_{\mathrm{c}+1}(\mathrm{~L})\right) . \\
& \text { Since } \operatorname{dim}\left(\mathrm{B} / \mathrm{B}^{2}\right) \leq \operatorname{dim}\left(\mathrm{L} / \mathrm{L}^{2}\right) \text {, we obtain: }
\end{aligned}
$$

Corollary 2.5. Under the assumptions and notation of the above Theorem, we have

$$
\operatorname{dim} M^{(c)}(L) \leq \operatorname{dim} M^{(c)}\left(L / L^{2}\right)+\operatorname{dim} L^{2}\left[\operatorname{dim}\left(L / L^{2}\right)\right]^{c}-\operatorname{dim} \gamma_{c+1}(L)
$$

Now, we compare our results to upper bound given [7], when $c=1$.

Theorem 2.6. Let $\mathrm{L}$ be a finite dimensional nilpotent Lie algebra of class $\mathrm{m}$ and $\mathrm{d}=\mathrm{d}(\mathrm{m})$. Then

$$
\operatorname{dim} M(L) \leq \sum_{\mathrm{k}=1}^{\mathrm{m}} \mathrm{l}_{\mathrm{d}}(\mathrm{k}+1)
$$

Example 2.7. Let $\mathrm{F}$ be a free Lie algebra on 2 generators and $\mathrm{L}=\mathrm{F} / \mathrm{F}^{3}$. Then $\mathrm{L}$ is a Lie algebra of 2 generators and class 2 . Thus $\operatorname{dim} \mathrm{L} / \mathrm{L}^{2}=\mathrm{l}_{2}(1)=2, \operatorname{dim} \mathrm{L}^{2} / \mathrm{L}^{3}=\mathrm{l}_{2}(2)=1$ and $\operatorname{dim} \mathrm{L}=3$. By Theorem 2.6,

$$
\operatorname{dim} M(L) \leq \sum_{j=1}^{2} 1_{2}(j+1)=1_{2}(2)+1_{2}(3)=1+\frac{1}{3}\left(\mu(1) 2^{3}+\mu(3) 2\right)=1+\frac{1}{3}(6)=3 .
$$

Note that $\mathrm{L}$ is a finite dimensional nilpotent Lie algebra of maximal class $(1+1)$ and $\mathrm{Z}(\mathrm{L})=\mathrm{L}^{2}$. By Corollary 1.4 and Lemma 1.3,

$$
\operatorname{dim} \mathrm{M}(\mathrm{L}) \leq \operatorname{dim} \mathrm{M}(\mathrm{L} / \mathrm{Z}(\mathrm{L}))+1=\operatorname{dim} \mathrm{M}\left(\mathrm{L} / \mathrm{L}^{2}\right)+1=1_{2}(2)+1=1+1=2 .
$$

Also, by Corollary 2.5,

$$
\operatorname{dim} \mathrm{M}(\mathrm{L}) \leq \operatorname{dim} \mathrm{M}\left(\mathrm{L} / \mathrm{L}^{2}\right)+\operatorname{dim} \mathrm{L}^{2}\left[\operatorname{dim}\left(\mathrm{L} / \mathrm{L}^{2}\right)\right]-\operatorname{dim} \mathrm{L}^{2}=\mathrm{l}_{2}(2)+1(2)-1=2 .
$$

Example 2.8. Let $\mathrm{F}$ be a free Lie algebra on 2 generators and
$\mathrm{L}=\mathrm{F} / \mathrm{F}^{4}$. Then $\mathrm{L}$ is a Lie algebra of 2 generators and class 3. Thus $\operatorname{dim} \mathrm{L}=5, \operatorname{dim} \mathrm{L}^{2}=3$ and $\operatorname{dim} \mathrm{L} / \mathrm{L}^{2}=1_{2}(1)=2$. By Theorem 2.6

$$
\begin{aligned}
& \operatorname{dim} M(L) \leq \sum_{j=1}^{3} l_{2}(j+1)=1_{2}(2)+l_{2}(3)+l_{2}(4) \\
& =1+2+\frac{1}{4}\left(\mu(1) 2^{4}+\mu(2) 2^{2}+\mu(4) 2\right) \\
& =1+\frac{1}{4}(16-4)=6
\end{aligned}
$$

Also, by Corollary 2.5,

$\operatorname{dim} \mathrm{M}(\mathrm{L}) \leq \operatorname{dim} \mathrm{M}\left(\mathrm{L} / \mathrm{L}^{2}\right)+\operatorname{dim} \mathrm{L}^{2}\left[\operatorname{dim}\left(\mathrm{L} / \mathrm{L}^{2}\right)\right]-\operatorname{dim} \mathrm{L}^{2}=\mathrm{l}_{2}(2)+3(2)-3=4$.

In this two examples, we see that our results give two better upper bounds for $\operatorname{dim}(\mathrm{L})$ than the previously known result.

\section{Acknowledgment}

The authors wish to thank Yazd Branch, Islamic Azad University for its support of research project under the title Verification of some properties of The c-nilpotent multiplier in Lie algebras.

\section{References}

1. Baer R (1945) Representations of groups as quotient groups, I, II, III. Trans Amer Math Soc 58: 295-419.

2. Burns J, Ellis G (1998) Inequalities for Baer invariants of finite groups Canad Math Bull 41: 385-391.

3. Leedham-Green CR, McKay S (1976) Baer-invariant, isologism, varietal laws and homology. Acta Math 137: 99-150

4. Batten P, Stitzinger E (1996) On covers of Lie algebras. Comm Alg 24: 4301 4317.

5. Batten P, Moneyhun K, Stitzinger E (1996) On characterizing nilpotent Lie algebras by their multipliers. Comm Alg 24: 4319-4330.

6. Salemkar AR, Edalatzadeh B, Araskhan M (2009) Some inequalities for the dimension of the c-nilpotent multiplier of Lie Algebras. J Algebra 322: 15751585.

7. Bahturin Y (1987) Identical relations in Lie algebras. VNU Science Press BV.
Citation: Sadeghieh A, Araskhan M (2015) Verification of Some Properties of the C-nilpotent Multiplier in Lie Algebras. J Generalized Lie Theory Appl 9: 228. doi:10.4172/1736-4337.1000228

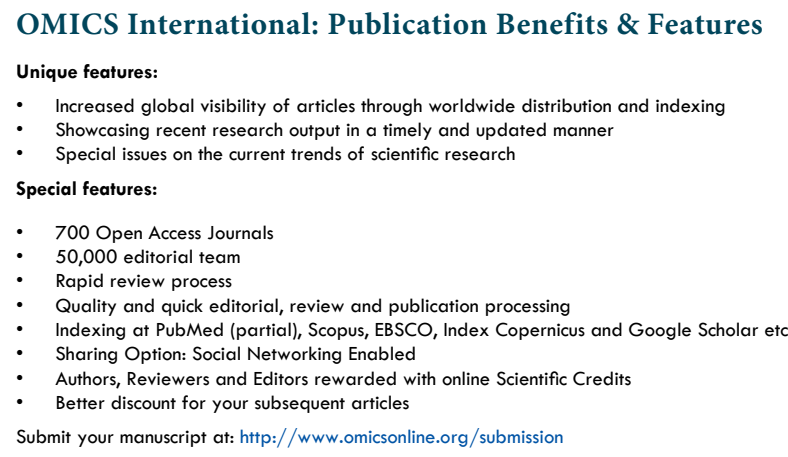

